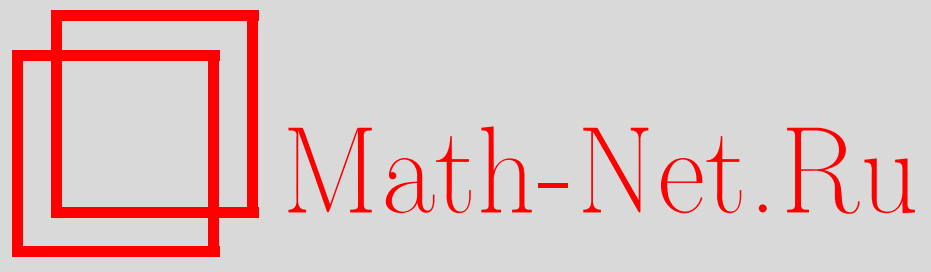

Р. А. Заторский, А. Р. Малярчук, Треугольные матрицы и комбинаторные формулы обращения, Матем. заметкu, 2009, том 85, выпуск 1, 12-21

DOI: https://doi.org/10.4213/mzm6585

Использование Общероссийского математического портала Math-Net.Ru подразумевает, что вы прочитали и согласны с пользовательским соглашением http://www . mathnet.ru/rus/agreement

Параметры загрузки:

IP : 54.210 .77 .194

26 апреля 2023 г., $15: 47: 34$

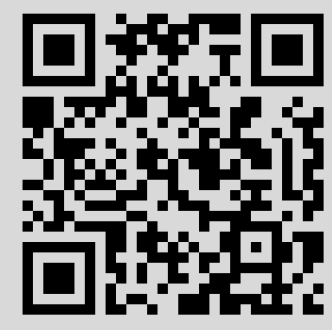


Том 85 выпуск 1 январь 2009

УДК 512.643

\section{Треугольные матрицы и комбинаторные формулы обращения}

\section{Р. А. Заторский, А.Р. Малярчук}

Работа посвящена построению матрицы, обратной к бесконечной треугольной матрице, установлению коэффициентов связи полиномиальных последовательностей и общих комбинаторных формул обращения.

Библиография: 6 названий.

\section{1. Треугольные матрицы и парадетерминанты.}

ОпредЕлЕниЕ 1 [1]. Треугольную таблицу чисел, принадлежащих полю $K$,

$$
A=\left(\begin{array}{cccc}
a_{11} & & & \\
a_{21} & a_{22} & & \\
\vdots & \vdots & \ddots & \\
a_{n 1} & a_{n 2} & \cdots & a_{n n}
\end{array}\right)_{n}
$$

назовем треугольной матрицей, а число $n$ - ее порядком.

Обратим внимание на тот факт, что треугольная матрица в нашем понимании не является матрицей в обычном смысле этого термина, поскольку она является треугольной, но не прямоугольной таблицей чисел.

Каждому элементу $a_{i j}$ матрицы $(1)$ поставим в соответствие $(i-j+1)$ элементов $a_{i k}, k=j, \ldots, i$, которые назовем производными элементами матрицы, порожденными ключевым элементом $a_{i j}$.

Произведение всех производных элементов, порожденных элементом $a_{i j}$, обозначим через $\left\{a_{i j}\right\}$ и назовем факториалъным произведением ключевого элемента $a_{i j}$, т.e.

$$
\left\{a_{i j}\right\}=\prod_{k=j}^{i} a_{i k}
$$

ОПРЕДЕЛЕНИЕ 2 [1]. Набор ключевых элементов матрицы (1) назовем нормальным набором этой матрицы, если они порождают монотрансверсаль, т.е. множество производных элементов мощности $n$, каждые два из которых не лежат в одном столбце этой матрицы.

(C) Р. А. ЗАторСКий, А.Р. МАлярчук, 2009 
Пусть $\mathbb{P}(n)$ - множество всех упорядоченных разбиений (композиций) (см. [2], [3; с. 67]) натурального числа $n$ на натуральные слагаемые. Известно, что

$$
|\mathbb{P}(n)|=\sum_{r=1}^{n}\left(\begin{array}{l}
n-1 \\
r-1
\end{array}\right)=2^{n-1} .
$$

Легко видеть, что между нормальными наборами ключевых элементов матрицы (1) и упорядоченными разбиениями натурального числа $n$ существует взаимно однозначное соответствие.

Каждому нормальному набору а ключевых элементов поставим в соответствие знак $(-1)^{\varepsilon(a)}$, где $\varepsilon(a)$ - сумма всех индексов ключевых элементов этого набора.

ОПРЕДЕЛЕНиЕ 3 [1].Парадетерминантом треугольной матрицы (1) назовем число

$$
\operatorname{ddet}(A)=\left\langle\begin{array}{cccc}
a_{11} & & \\
a_{21} & a_{22} & \\
\vdots & \vdots & \ddots & \\
a_{n 1} & a_{n 2} & \cdots & a_{n n}
\end{array}\right\rangle=\sum_{\left(\alpha_{1}, \alpha_{2}, \ldots, \alpha_{r}\right) \in \mathbb{P}(n)}(-1)^{\varepsilon(a)} \prod_{s=1}^{r}\left\{a_{i(s), j(s)}\right\},
$$

где $a_{i(s), j(s)}-$ ключевой элемент, соответствующий $s$-й компоненте разбиения $\alpha=$ $\left(\alpha_{1}, \alpha_{2}, \ldots, \alpha_{r}\right)$.

Каждому элементу $a_{i j}$ треугольной матрицы (1) поставим в соответствие треугольную таблицу $R_{i j}$ элементов этой матрицы, полученную вычеркиванием строк и столбцов, расположенных ниже и левее этого элемента, и назовем ее углом этой матрицы.

ОПРЕДЕЛЕНИЕ 4 [1].Прямоугольную таблицу элементов треугольной матрицы (1) назовем вписанной в эту матрицу, если одна ее вершина совпадает с элементом $a_{n 1}$, а противоположная к ней - с элементом $a_{i i}, i=1,2, \ldots, n$. Обозначим эту таблицу через $T(i)$.

ПримечАниЕ 1. Если в определении 4 значение индекса $i$ равно 1 или $i=n$, то прямоугольная таблица вырождается соответственно в первый столбец или последнюю строку.

При вычислении парадетерминанта удобно пользоваться алгебраическими дополнениями.

ОПРЕДЕЛЕНИЕ 5 [1]. Алгебраическим дополнением $D_{i j}$ к факториальному произведению $\left\{a_{i j}\right\}$ ключевого элемента $a_{i j}$ треугольной матрицы (1) назовем число

$$
D_{i j}=(-1)^{i+j} \cdot \operatorname{ddet}\left(R_{j-1,1}\right) \cdot \operatorname{ddet}\left(R_{n, i+1}\right),
$$

где $R_{j-1,1}$ и $R_{n, i+1}-$ углы этой матрицы.

Хотя определение парадетерминанта треугольной матрицы существенно отличается от классического понятия детерминанта, их свойства во многом аналогичны. 
ТЕоРема 1 (Разложение парадетерминанта по элементам вписанной прямоугольной таблицы, [1]). Пусть $A$ - треугольная матрица (1) и T(i) - некоторая вписанная прямоугольная таблица элементов этой матрицы. Тогда справедливо равенство

$$
\operatorname{ddet}(A)=\sum_{s=1}^{i} \sum_{r=i}^{n}\left\{a_{r s}\right\} \cdot D_{r s},
$$

где $D_{r s}$ - алгебрачческое дополнение $\kappa$ факториальному произведению ключевого элемента $a_{r s}$, который принадлежит вписанной прямоугольной таблище $T(i)$.

СЛЕДСТВИЕ 1 [1]. При $i=1$ по формуле (3) и примечанию 1 получим разложение парадетерминанта по элементам первого столбиа

$$
\operatorname{ddet}(A)=\sum_{r=1}^{n}(-1)^{r+1} \cdot\left\{a_{r 1}\right\} \cdot \operatorname{ddet}\left(R_{n, r+1}\right) .
$$

Если $i=n$, то получим разложение по элементам последней строки

$$
\operatorname{ddet}(A)=\sum_{s=1}^{n}(-1)^{n+s} \cdot\left\{a_{n s}\right\} \cdot \operatorname{ddet}\left(R_{s-1,1}\right) .
$$

Более детальную информацию о треугольных матрицах и парадетерминантах можно найти в [1], [4].

2. Полиномиальные последовательности и треугольные матрицы. Обозначим через $P$ множество всех вещественных многочленов одной переменной.

ОПРЕДЕЛЕНИЕ 6 [5]. Полиномиальная последовательность - семейство $\left\{p_{n}(x)\right.$ : $\left.n \in \mathbb{N}_{0}\right\}$ таких вещественных многочленов, что $\operatorname{deg}\left(p_{n}(x)\right)=n$ для всех $n$.

Так как многочлены полиномиальной последовательности имеют возрастающую степень, они образуют базис в линейном пространстве $P$. Полиномиальную последовательность $\left\{1, x, x^{2}, \ldots\right\}$ назовем стандартной.

Иногда полиномиальную последовательность мы будем представлять в виде вектора-столбца

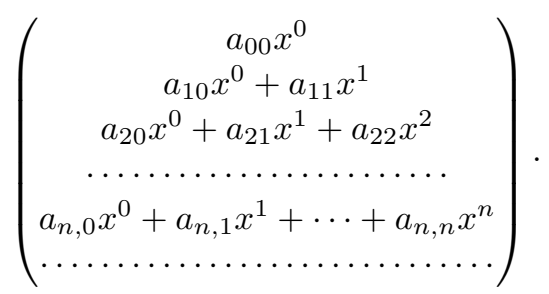

Тут согласно определению 6 имеют место неравенства $a_{i i} \neq 0, i=0,1,2, \ldots$.

Определим сумму двух полиномиальных последовательностей и умножение полиномиальной последовательности на любое действительное число аналогично операции суммы векторов и умножения вектора на число.

Две полиномиальные последовательности будем считать равными, если равны их соответствующие компоненты. 
Пусть заданы две полиномиальные последовательности

$$
\begin{gathered}
f=\left(\begin{array}{c}
f_{0} \\
f_{1} \\
f_{2} \\
\vdots
\end{array}\right)=\left(\begin{array}{c}
f_{00} \\
f_{10}+f_{11} x \\
f_{20}+f_{21} x+f_{22} x^{2} \\
\vdots
\end{array}\right), \\
g=\left(\begin{array}{c}
g_{0} \\
g_{1} \\
g_{2} \\
\vdots
\end{array}\right)=\left(\begin{array}{c}
g_{00} \\
g_{10}+g_{11} x \\
g_{20}+g_{21} x+g_{22} x^{2} \\
\vdots
\end{array}\right) .
\end{gathered}
$$

Тогда в силу неравенств $f_{i i} \neq 0, i=0,1,2, \ldots$, многочлен $g_{s}$ единственным образом можно представить в виде линейной комбинации многочленов $f_{0}, f_{1}, \ldots, f_{s}$.

Таким образом, треугольную матрицу вида

$$
\left(\begin{array}{cccc}
a_{00} & & & \\
a_{10} & a_{11} & & \\
a_{20} & a_{21} & a_{22} & \\
\vdots & \vdots & \vdots & \ddots
\end{array}\right)
$$

можно, например, интерпретировать как линейное преобразование $A$, которое полиномиальной последовательности $f$ ставит в соответствие полиномиальную последовательность $g$ :

$$
\left\{\begin{array}{l}
g_{0}=a_{00} f_{0}, \\
g_{1}=a_{10} f_{0}+a_{11} f_{1}, \\
g_{2}=a_{20} f_{0}+a_{21} f_{1}+a_{22} f_{2}, \\
\ldots \ldots \ldots \ldots \ldots \ldots \ldots \ldots \ldots \ldots
\end{array}\right.
$$

Определим матричный вид системы уравнений (7) следующим образом:

$$
\left(\begin{array}{c}
g_{0} \\
g_{1} \\
g_{2} \\
\vdots
\end{array}\right) \stackrel{\text { def }}{=}\left(\begin{array}{cccc}
a_{00} & & & \\
a_{10} & a_{11} & & \\
a_{20} & a_{21} & a_{22} & \\
\vdots & \vdots & \vdots & \ddots
\end{array}\right) \cdot\left(\begin{array}{c}
f_{0} \\
f_{1} \\
f_{2} \\
\vdots
\end{array}\right)
$$

ПримЕЧАНИЕ 2. Если полиномиальную последовательность рассматривать как некоторый бесконечномерный вектор, то линейное преобразование (7) становится частным случаем общего линейного преобразования бесконечномерного векторного пространства. Поэтому справедливо равенство $(A B) C=A(B C)$ и неравенство $A B \neq B A$.

Очевидно, что выполняется также равенство

$$
A E=E A,
$$

где $E$ - единичная треугольная матрица. 
Наконец отметим, что произведение двух треугольных матриц с ненулевыми диагональными элементами дает треугольную матрицу с ненулевыми диагональными элементами.

Найдем обратную треугольную матрицу $A^{-1}$ к треугольной матрице $A$. С этой целью решим систему уравнений (7) относительно системы неизвестных $\left(f_{0}, f_{1}, f_{2}, \ldots\right)$. Покажем, что решением этой системы уравнений является

$$
f_{i}=\sum_{j=0}^{i} \frac{(-1)^{i+j}}{a_{j j}} \cdot\left\langle\frac{a_{r+j+1, s+j}}{a_{r+j+1, s+j+1}}\right\rangle_{0 \leqslant s \leqslant r \leqslant i-j-1} \cdot g_{j}, \quad i=0,1, \ldots
$$

При $i=0$ система уравнений (7) имеет решение

$$
f_{0}=\sum_{j=0}^{0} \frac{(-1)^{0+j}}{a_{00}} \cdot\left\langle\frac{a_{r+j+1, s+j}}{a_{r+j+1, s+j+1}}\right\rangle_{0 \leqslant s \leqslant r \leqslant-j-1} \cdot g_{j}=\frac{1}{a_{00}} \cdot g_{0} ;
$$

тут мы считаем, что

$$
\left\langle\frac{a_{r+j+1, s+j}}{a_{r+j+1, s+j+1}}\right\rangle_{0 \leqslant s \leqslant r \leqslant-1}=1 .
$$

Предположим, что система уравнений (7) при $i=k-1$ имеет решение

$$
f_{k-1}=\sum_{j=0}^{k-1} \frac{(-1)^{k-1+j}}{a_{j j}} \cdot\left\langle\frac{a_{r+j+1, s+j}}{a_{r+j+1, s+j+1}}\right\rangle_{0 \leqslant s \leqslant r \leqslant k-j-2} \cdot g_{j},
$$

и докажем, что при этом

$$
f_{k}=\sum_{j=0}^{k} \frac{(-1)^{k+j}}{a_{j j}} \cdot\left\langle\frac{a_{r+j+1, s+j}}{a_{r+j+1, s+j+1}}\right\rangle_{0 \leqslant s \leqslant r \leqslant k-j-1} \cdot g_{j} .
$$

Найдем из последнего уравнения системы (7) при $i=k$ многочлен $f_{k}$ :

$$
f_{k}=-\sum_{i=0}^{k-1} \frac{a_{k i}}{a_{k k}} f_{i}+\frac{1}{a_{k k}} g_{k}
$$

Возьмем в последнем равенстве вместо многочленов $f_{i}, i=0,1, \ldots, k-1$, их известные значения (8):

$$
f_{k}=-\sum_{i=0}^{k-1} \frac{a_{k i}}{a_{k k}} \sum_{j=0}^{i} \frac{(-1)^{i+j}}{a_{j j}} \cdot\left\langle\frac{a_{r+j+1, s+j}}{a_{r+j+1, s+j+1}}\right\rangle_{0 \leqslant s \leqslant r \leqslant i-j-1} \cdot g_{j}+\frac{1}{a_{k k}} g_{k} .
$$

Поменяем порядок суммирования:

$$
f_{k}=\sum_{j=0}^{k-1}(-1)^{k+j} \frac{1}{a_{j j}}\left(\sum_{i=j}^{k-1}(-1)^{i-k+1} \frac{a_{k i}}{a_{k k}} \cdot\left\langle\frac{a_{r+j+1, s+j}}{a_{r+j+1, s+j+1}}\right\rangle_{0 \leqslant s \leqslant r \leqslant i-j-1}\right) \cdot g_{j}+\frac{1}{a_{k k}} g_{k} .
$$

Но выражение в скобках в последнем равенстве является разложением парадетерминанта

$$
\left\langle\frac{a_{r+j+1, s+j}}{a_{r+j+1, s+j+1}}\right\rangle_{0 \leqslant s \leqslant r \leqslant k-j-1}
$$


по элементам последней строки, поэтому имеем

$$
\begin{array}{r}
f_{k}=\sum_{j=0}^{k-1}(-1)^{k+j} \frac{1}{a_{j j}} \cdot\left\langle\frac{a_{r+j+1, s+j}}{a_{r+j+1, s+j+1}}\right\rangle_{0 \leqslant s \leqslant r \leqslant k-j-1} \cdot g_{j}+\frac{1}{a_{k k}} g_{k} \\
=\sum_{j=0}^{k}(-1)^{k+j} \frac{1}{a_{j j}} \cdot\left\langle\frac{a_{r+j+1, s+j}}{a_{r+j+1, s+j+1}}\right\rangle_{0 \leqslant s \leqslant r \leqslant k-j-1} \cdot g_{j} .
\end{array}
$$

Таким образом, нами доказана следующая

ТЕОРема 2. Обратной треугольной матрищей $\kappa$ матрице (6) является треугольная матрица

$$
\left(b_{i j}\right)_{0 \leqslant j \leqslant i<\infty}=\left(\frac{(-1)^{i+j}}{a_{j j}} \cdot\left\langle\frac{a_{r+j+1, s+j}}{a_{r+j+1, s+j+1}}\right\rangle_{0 \leqslant s \leqslant r \leqslant i-j-1}\right)_{0 \leqslant j \leqslant i<\infty} .
$$

Следует отметить, что парадетерминант

$$
\left\langle\frac{a_{r+j+1, s+j}}{a_{r+j+1, s+j+1}}\right\rangle_{0 \leqslant s \leqslant r \leqslant i-j-1}
$$

определен даже в тех случаях, когда знаменатель выражения $a_{r+j+1, s+j} / a_{r+j+1, s+j+1}$ обращается в нуль, ибо при нахождении значения парадетерминанта нули сокращаются и неопределенности исчезают.

3. Коэффициенты связи полиномиальных последовательностей. В [5; с. 124] отмечается, что задача определения коэффициентов связи для произвольных полиномиальных последовательностей представляет значительные трудности и выделяется класс так называемых биномиальных последовательностей, для которых эта задача поддается разрешению.

Определим коэффициенты связи для произвольных полиномиальных последовательностей.

Найдем матрицу линейного преобразования полиномиальной последовательности $f$, задающейся равенством (4), в полиномиальную последовательность $g$, которая определена равенством (5). С этой целью найдем матрицу $A$ из матричного равенства

$$
\left(\begin{array}{cccc}
a_{00} & & & \\
a_{10} & a_{11} & & \\
a_{20} & a_{21} & a_{22} & \\
\vdots & \vdots & \vdots & \ddots
\end{array}\right) \cdot\left(\begin{array}{cccc}
f_{00} & & & \\
f_{10} & f_{11} & & \\
f_{20} & f_{21} & f_{22} & \\
\vdots & \vdots & \vdots & \ddots
\end{array}\right) \cdot\left(\begin{array}{c}
1 \\
x \\
x^{2} \\
\vdots
\end{array}\right)=\left(\begin{array}{cccc}
g_{00} & & & \\
g_{10} & g_{11} & & \\
g_{20} & g_{21} & g_{22} & \\
\vdots & \vdots & \vdots & \ddots
\end{array}\right) \cdot\left(\begin{array}{c}
1 \\
x \\
x^{2} \\
\vdots
\end{array}\right)
$$

или

$$
A F(X)=G(X)
$$

где $(X)$ обозначает стандартную полиномиальную последовательность.

Из последнего равенства очевидно следуют равенства

$$
A F=G \quad \text { и } \quad A=G F^{-1} .
$$


Умножая матрицы $G$ и $F^{-1}$, получим

$$
\begin{aligned}
a_{i j} & =\sum_{k=j}^{i} g_{i k} \cdot\left(\frac{(-1)^{k+j}}{f_{j j}} \cdot\left\langle\frac{f_{r+j+1, s+j}}{f_{r+j+1, s+j+1}}\right\rangle_{0 \leqslant s \leqslant r \leqslant k-j-1}\right) \\
& =\frac{(-1)^{i+j}}{f_{j j}} \sum_{k=j}^{i}(-1)^{i-k} g_{i k} \cdot\left\langle\frac{f_{r+j+1, s+j}}{f_{r+j+1, s+j+1}}\right\rangle_{0 \leqslant s \leqslant r \leqslant k-j-1} .
\end{aligned}
$$

Но сумма в правой части равенства (9) является разложением парадетерминанта по элементам последней строки

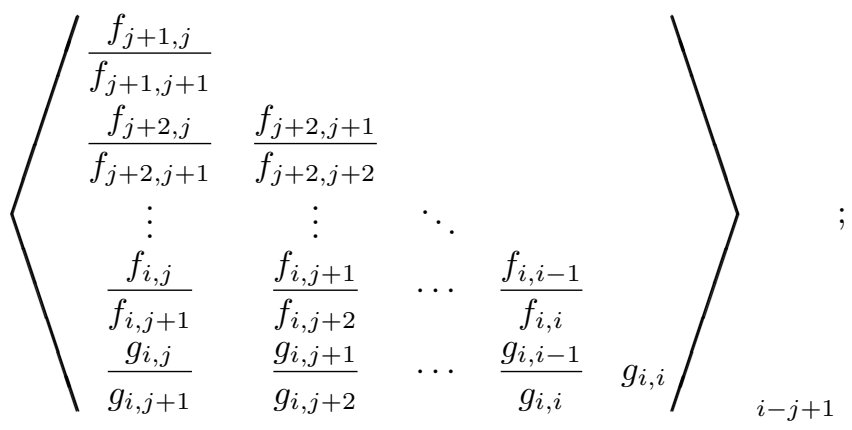

следовательно, нами доказана следующая

ТЕОРема 3. Элементы матрицы линейного преобразования полиномиальной последовательности $f=F(X)$, которая задается равенством (4), в полиномиальную последовательность $g=G(X)$, задающуюся равенством (5), равны

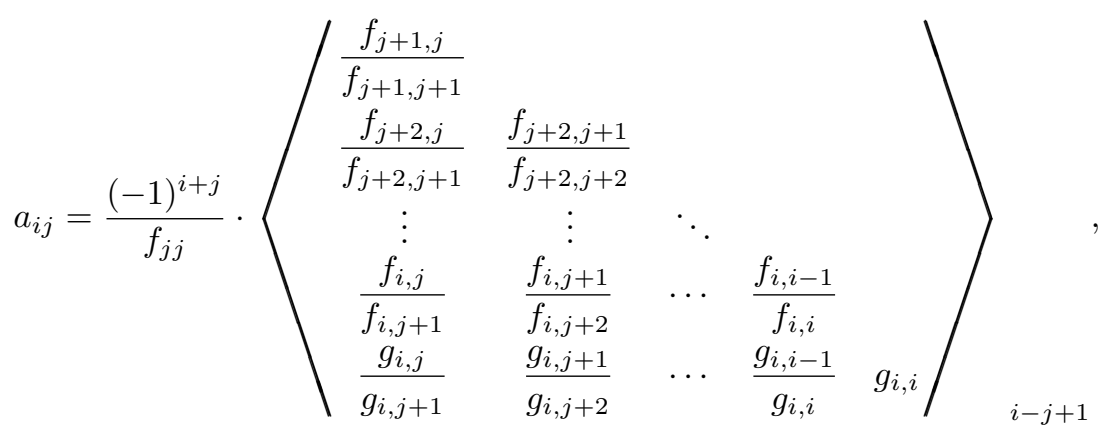

$0 \leqslant j \leqslant i<\infty$.

4. Формулы обращения. В [6; с.7-8] Риордан отмечает, что “... число различных пар взаимно обратных соотношений пока невелико, а возможности для дальнейшего увеличения их числа определить трудно".

Теперь в нашем распоряжении есть все необходимые сведения для того, чтобы доказать следующую, важную для комбинаторного анализа теорему.

ТеоремА 4 (теорема об обращении). Пусть заданы две треугольные матрицы

$$
F=\left(\begin{array}{cccc}
f_{00} & & & \\
f_{10} & f_{11} & & \\
f_{20} & f_{21} & f_{22} & \\
\vdots & \vdots & \vdots & \ddots
\end{array}\right) \quad u \quad G=\left(\begin{array}{cccc}
g_{00} & & & \\
g_{10} & g_{11} & & \\
g_{20} & g_{21} & g_{22} & \\
\vdots & \vdots & \vdots & \ddots
\end{array}\right)
$$


с ненулевыми диагональными элементами. Тогда справедливо обращение

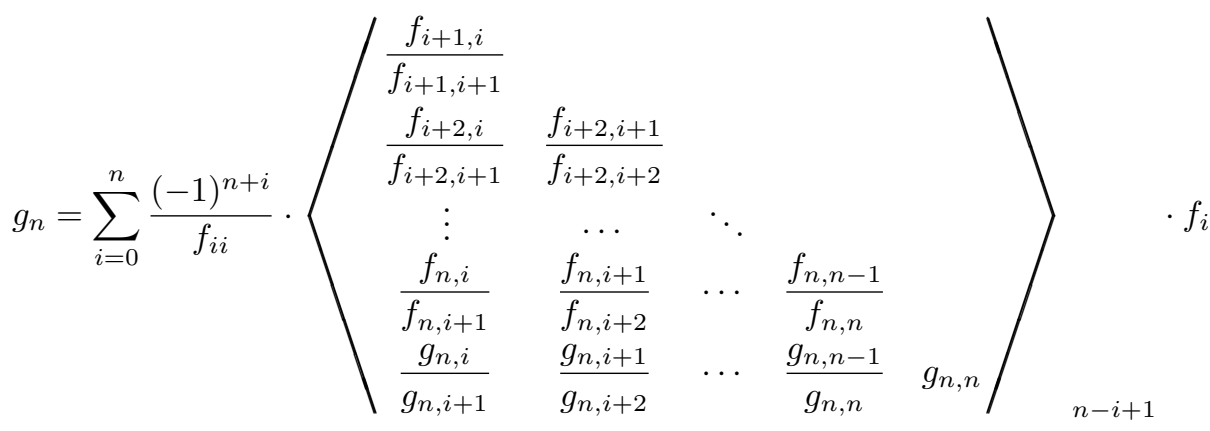

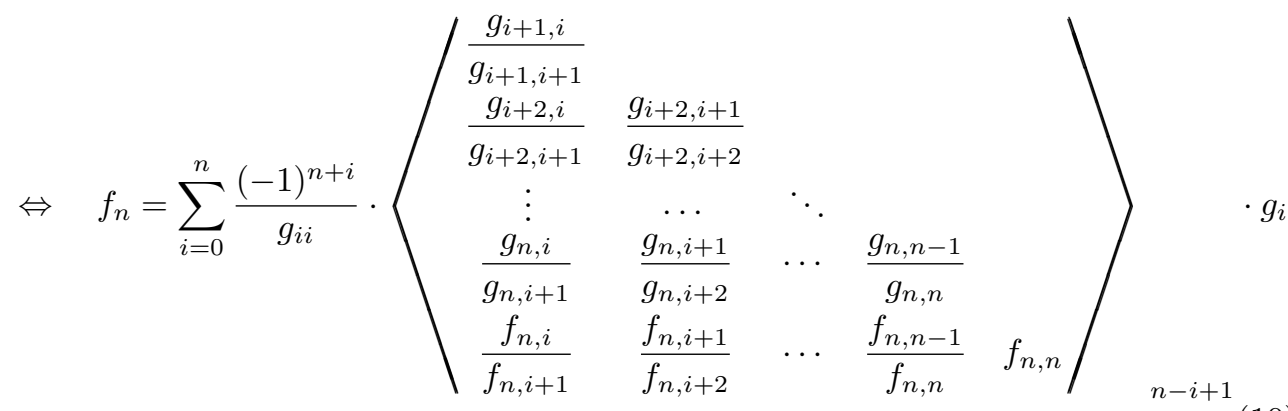

для $n \in \mathbb{N}_{0}$, где $f_{i}, g_{i}, i=0,1, \ldots, n,-$ произвольные действительные числа.

ДокАЗАТЕЛЬСтво. Треугольные матрицы $G F^{-1}$ и $F G^{-1}$ в равенствах $G(X)=$ $G F^{-1} F(X)$ и $F(X)=F G^{-1} G(X)$ можно найти при помощи теоремы 3 ; поэтому сравнивая в этих матричных равенствах последние компоненты, получим обращение (10). Остается отметить, что поскольку матрицы $G F^{-1}$ и $F G^{-1}$ взаимно обратны, это обращение не нарушится, если вместо многочленов $f_{i}, g_{i}, i=0,1, \ldots, n$, положить соответственно любые действительные числа.

СлеДСтвиЕ 2. Пусть $F=E=\left(\delta_{i j}\right)_{0 \leqslant j \leqslant i<\infty} u$

$$
G=\left(\begin{array}{cccc}
g_{00} & & & \\
g_{10} & g_{11} & & \\
g_{20} & g_{21} & g_{22} & \\
\vdots & \vdots & \vdots & \ddots
\end{array}\right)
$$

Тогда справедливо обращение

$$
g_{n}=\sum_{i=0}^{n} g_{n, i} f_{i} \quad \Leftrightarrow \quad f_{n}=\sum_{s=0}^{n} \frac{(-1)^{n-s}}{g_{s, s}} \operatorname{ddet}\left(\frac{g_{s+i, s+j-1}}{g_{s+i, s+j}}\right)_{1 \leqslant j \leqslant i \leqslant n-s} \cdot g_{s} .
$$

Если, в частности, в следствии 2, элементы матрицы $G$ совпадают с биномиальными коэффициентами, числами Стирлинга первого рода, числами Ла со знаком, то формулы (11) дадут соответственно биномиальное обращение, обращение Стирлинга или обращение Ла (см. [5; с. 120]). 
Приведем примеры новых формул обращения.

Матрице

$$
\left(\begin{array}{ccccccc}
1 & & & & & & \\
1 & 1 & & & & & \\
2 & 1 & 1 & & & & \\
3 & 2 & 1 & 1 & & & \\
5 & 3 & 2 & 1 & 1 & & \\
8 & 5 & 3 & 2 & 1 & 1 & \\
13 & 8 & 5 & 3 & 2 & 1 & 1
\end{array}\right)
$$

соответствует обратная треугольная матрица

$$
\left(\begin{array}{cccccccc}
1 & & & & & & \\
-1 & 1 & & & & & \\
-1 & -1 & 1 & & & & \\
0 & -1 & -1 & 1 & & & \\
0 & 0 & -1 & -1 & 1 & & \\
0 & 0 & 0 & -1 & -1 & 1 & \\
0 & 0 & 0 & 0 & -1 & -1 & 1
\end{array}\right)
$$

Поэтому мы имеем формулу обращения

$$
v_{n}=\sum_{i=0}^{n} F_{n-i} u_{i} \quad \Leftrightarrow \quad u_{n}=v_{n}-v_{n-1}-v_{n-2}
$$

где $F_{i}, i=0,1, \ldots,-$ числа Фибоначчи.

Пусть

$$
A=\left((-1)^{[(i-j) / 2]}\right)_{0 \leqslant j \leqslant i \leqslant n},
$$

тогда

$$
A^{-1}=\left(\begin{array}{ccccccc}
1 & & & & & & \\
-1 & 1 & & & & & \\
2 & -1 & 1 & & & & \\
-2 & 2 & -1 & 1 & & & \\
\vdots & \vdots & \vdots & \vdots & \ddots & & \\
(-1)^{n-2} 2 & (-1)^{n-3} 2 & (-1)^{n-4} 2 & (-1)^{n-5} 2 & \cdots & 1 & \\
(-1)^{n-1} 2 & (-1)^{n-2} 2 & (-1)^{n-3} 2 & (-1)^{n-4} 2 & \cdots & -1 & 1
\end{array}\right)_{n}
$$

Этой паре соответствует следующая формула обращения:

$$
v_{n}=\sum_{i=0}^{n}(-1)^{[(n-i) / 2]} u_{i} \quad \Leftrightarrow \quad u_{n}=v_{n}-v_{n-1}+2 \sum_{i=0}^{n-2}(-1)^{i} v_{i} .
$$

\section{СПИСОК ЦИТИРОВАННОЙ ЛИТЕРАТУРЫ}

[1] R. A. Zators'kiŭ, "On paradeterminants and parapermanents of triangular matrices", Mat. Stud., 17:1 (2002), 3-17. 
[2] Р. Стенли, Перечислительная комбинаторика, Мир, М., 1990.

[3] Г. Эндрюс, Теория разбиений, Наука, М., 1982.

[4] Р.А. Заторский, "Определители треугольных матриц и траектории на диаграммах Ферре", Матем. заметки, 72:6 (2002), 834-852.

[5] М. Айгнер, Комбинаторная теория, Мир, М., 1982.

[6] Дж. Риордан, Комбинаторные тождества, Наука, М., 1982.

Р. А. Заторский

E-mail: romazz@rambler.ru

\section{А. Р. Малярчук}

Прикарпатский национальный университет им. В. Стефаника 